\title{
Wavelet Adaptive Algorithm and Its Application to MRE Noise Control System
}

\author{
Zhang Yulin and Zhao Xiuyang \\ School of Information Science and Engineering, University of Jinan, Jinan 250022, China \\ Correspondence should be addressed to Zhao Xiuyang; zhaoxy@ujn.edu.cn \\ Received 24 January 2015; Accepted 21 March 2015 \\ Academic Editor: Seung-Bok Choi
}

Copyright ( 2015 Z. Yulin and Z. Xiuyang. This is an open access article distributed under the Creative Commons Attribution License, which permits unrestricted use, distribution, and reproduction in any medium, provided the original work is properly cited.

To address the limitation of conventional adaptive algorithm used for active noise control (ANC) system, this paper proposed and studied two adaptive algorithms based on Wavelet. The twos are applied to a noise control system including magnetorheological elastomers (MRE), which is a smart viscoelastic material characterized by a complex modulus dependent on vibration frequency and controllable by external magnetic fields. Simulation results reveal that the Decomposition LMS algorithm (D-LMS) and Decomposition and Reconstruction LMS algorithm (DR-LMS) based on Wavelet can significantly improve the noise reduction performance of MRE control system compared with traditional LMS algorithm.

\section{Introduction}

The most popular algorithm used to adapt FIR filters is the Widrow-Hoff LMS [1], which is shown in Figure 1. Its popularity is due to its low computational complexity and robustness to implementation errors.

As in Figure 1, $x(n), d(n), e(n)$, and $y(n)$ denote the reference of system, disturbance, control error, and control signal, respectively. The system control signal can be expressed as

$$
y(n)=X^{T}(n) W(n),
$$

where $W(n)=\left[w_{0}, w_{1}, \ldots, w_{N-1}\right]^{T}$ is the weight vector of the $N$ order control filter, $X^{T}(n)=x(n) \sum_{i=0}^{N-1} z^{-i}$, and $T$ denotes the vectors transpose. The goal is to minimize the output error:

$$
e(n)=d(n)-y(n) .
$$

According to the Widrow-Hoff LMS algorithm [1], the weight of control filter can be adjusted by

$$
W(n+1)=W(n)+2 \mu X(n) e(n),
$$

where $\mu$ is the step-size parameter. The convergence rate of this algorithm depends on the condition numbers of the autocorrelation $R_{x}$ of the reference signal. When the eigenvalues of $R_{x}$ are widely spread, the excess mean square error produced by LMS algorithm is primarily determined by the largest eigenvalue, and the time taken by the average tapweight vector $E[\widehat{W}(k)]$ to converge is limited by the smallest eigenvalue. However, the speed of convergence of the mean square error is affected by the spread of the eigenvalues of $R_{x}$.

The speed of convergence of the LMS algorithm may slow down when the correlation matrix of the inputs is ill-conditioned, which implies that the control system with Widrow-Hoff LMS adaptive algorithm might become unstable when inputs change indefinitely. In order to enhance the performance of the algorithm, Wavelet Transform is proposed in this study due to its time-frequency localization $[2,3]$. This paper presents two kinds of adaptive wavelet algorithm: Decomposition LMS algorithm (D-LMS) and Decomposition and Reconstruction LMS algorithm (DRLMS).

\section{Decomposition LMS Algorithm (D-LMS)}

In D-LMS algorithm, the input signal is decomposed into various wavelet spaces according to various scales to form the input vector, and then the adaptive filter weight 


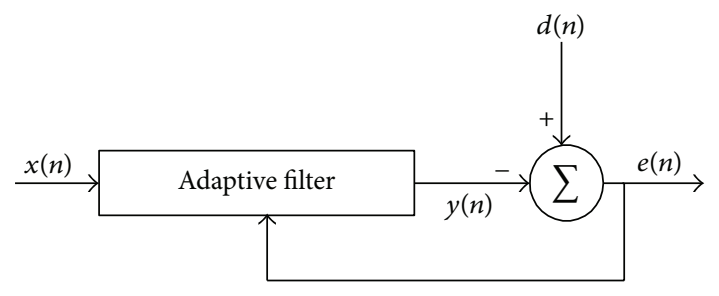

FIGURE 1

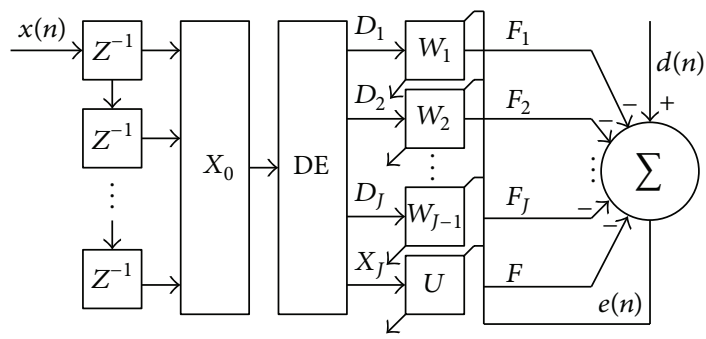

FIgURE 2: Decomposition LMS algorithm.

corresponding to a certain frequency component is adjusted. This means that the proportion of each frequency of input signal is altered so as to reconstruct the output signal to approximate the desired signal [4-6].

2.1. Structure of D-LMS. Schematic of the D-LMS is provided in Figure 2. Here, $x(n)$ is the input signal, $X_{0}=$ $x(n) \sum_{i=0}^{N-1} z^{-i}$ represents the input vector of the D-LMS algorithm, and $D E$ denotes the signal decomposition with MALLAT algorithm [7]. $D_{j}=\left[d_{j, 0}, d_{j, 1}, \ldots, d_{j, 2^{-j} N-1}\right]^{T}(j=$ $1, \ldots, J)$ is the $j$ th level detail signal of original signal, $X_{J}=$ $\left[x_{0}, x_{1}, \ldots, x_{2^{-j} N-1}\right]^{T}$ is the $J$ th level approximation signal of original signal, $W_{j}=\left[w_{j, 0}, w_{j, 1}, \ldots, w_{j, 2^{-j} N-1}\right]^{T}(j=$ $1, \ldots, J)$ is the weight vector of $j$ th level details signal, and $U=\left[u_{0}, u_{1}, \ldots, u_{2^{-J} N-1}\right]^{T}$ is weight vector of the $J$ th level approximation signal.

2.2. Adaptation of Weight Vectors. As seen in Figure 2,

$$
\begin{gathered}
F_{j}=D_{j}^{T} W_{j} \quad(j=1, \ldots, J), \\
F=X_{J}^{T} U \\
e(n)=d_{n}-\sum_{j=1}^{J} F_{j}-F
\end{gathered}
$$

Equation (6) also can be written as follows:

$$
e(n)=d_{n}-\sum_{j=1}^{J} D_{j}^{T} W_{j}-X_{J} U
$$

According to the LMS algorithm, the equations of $W$ and $U$ are as follows:

$$
\begin{gathered}
W_{j}=W_{j}+\mu_{j}\left(-\widehat{\nabla}_{j}\right) \quad(j=1, \ldots, J), \\
U=U+\mu\left(-\widehat{\nabla}_{J}\right)
\end{gathered}
$$

where $\mu_{j}(j=1, \ldots, J)$ and $\mu$ represent the step-size of the $j$ th level detail signal and $J$ th level approximation signal, respectively. $\widehat{\nabla}_{j}$ and $\widehat{\nabla}_{J}$, which denote the instantaneous estimation gradient vectors with $e^{2}(n)$ to $W_{j}$ and $U$ separately, can be expressed as follows:

$$
\begin{gathered}
\widehat{\nabla}_{j}=2 e(n) \frac{\partial e(n)}{\partial W_{j}} \quad(j=1, \ldots, J) \\
\widehat{\nabla}_{J}=2 e(n) \frac{\partial e(n)}{\partial U} .
\end{gathered}
$$

In this study, we can place the emphasis only on the adjustment of $W_{j}$ as $U$ resembles $W_{j}$ in the adjustment way.

First, rewrite (10) as follows:

$$
\begin{gathered}
\widehat{\nabla}_{j}=2 e(n)\left[\frac{\partial e(n)}{\partial w_{j, 0}}, \frac{\partial e(n)}{\partial w_{j, 1}}, \ldots, \frac{\partial e(n)}{\partial w_{j, 2^{-j} N-1}}\right]^{T}, \\
\widehat{\nabla}_{J}=2 e(n)\left[\frac{\partial e(n)}{\partial u_{0}}, \frac{\partial e(n)}{\partial u_{1}}, \ldots, \frac{\partial e(n)}{\partial u_{2^{-J} N-1}}\right]^{T} .
\end{gathered}
$$

According to (4) and (5), the following equation is obtained:

$$
\frac{\partial e(n)}{\partial w_{i, k}}=-\sum_{j=0}^{J} D_{j}^{T} \frac{\partial W_{j}}{\partial w_{i, k}}-X_{J}^{T} \frac{\partial U}{\partial w_{i, k}}=-d_{i, k} .
$$

Then, the adaptation of $W$ can be given by

$$
w_{j, k}=w_{j, k}+2 \mu_{j} e(n) d_{j, k} .
$$

Here, $i=1, \ldots, J, k=0, \ldots, 2^{-i} N-1$. Similarly, the adaptation of $U$ can be expressed by

$$
u_{k}=u_{k}+2 u e(n) x_{k} \text {. }
$$

Here, $0 \leq k<2^{-J} N$.

\section{Decomposition and Reconstruction LMS Algorithm (DR-LMS)}

3.1. The Structure of DR-LMS. Schematic of the DR-LMS algorithm is shown in Figure 3, in which the signal is not only decomposed, but also reconstructed.

Where $X_{0}, D E, D_{j}(j=1, \ldots, J), X_{J}, W_{j}$, and $U$ are the same meanings as in Figure 2. $V_{j}(j=1, \ldots, J-1)$ and $Y_{J}$ are the vectors, which can be expressed, respectively, by

$$
\begin{gathered}
V_{j}=D_{j} \widehat{\times} W_{j} \quad j=1, \ldots, J, \\
Y_{J}=X_{J} \widehat{\times} U .
\end{gathered}
$$




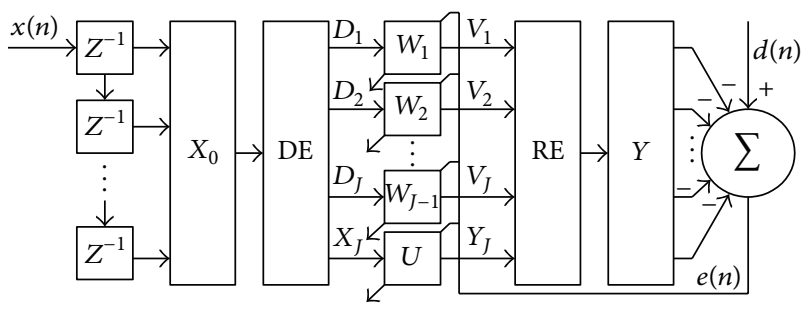

FIGURE 3: DR-LMS algorithm.

Here, $\widehat{x}$ denotes that each component of vector is multiplied by corresponding component of another vector. $R E$ signifies the signal reconstruction. $Y=\left[y_{0}, y_{1}, \ldots, y_{N-1}\right]^{T}$ represents the results of reconstruction.

It is obvious that the DR-LMS algorithm consists of three parts: firstly, the signal is decomposed into various levels, then each level detail signal and final approximation signal are multiplied by the corresponding weight vectors, and, finally, these signals are reconstructed by MALLAT algorithm to form the output of DR-LMS algorithm. In the process, the weight vectors are adapted to minimize the $e(n)$; therefore, the law of the adaptation is crucial.

3.2. Adaptation of Weight Vector. As indicated in Figure 3, the error $e(n)$ is represented by

$$
e(n)=d(n)-Y^{T} T,
$$

where $T=[1,1, \ldots, 1]_{1 \times N}^{T}$. According to LMS algorithm, in order to minimize the error $e(n), W_{j}$ and $U$ can be adapted by (8) and (9). With the foregoing discussion, we only study the adaptation way of $W_{j}$ and then can obtain the adaptation way of $U$ in the same way.

According to MALLAT algorithm and Figure 3, the following equations are obtained:

$$
\begin{aligned}
Y(m)= & 2 \sum_{k} 2 h(m-2 k) Y_{1}(k) \\
& +2 \sum_{k} g(m-2 k) V_{1}(k), \\
Y_{1}(m)= & 2 \sum_{k} h(m-2 k) Y_{2}(k) \\
& +2 \sum_{k} g(m-2 k) V_{2}(k), \\
Y_{2}(m)= & 2 \sum_{k} h(m-2 k) Y_{3}(k) \\
& +2 \sum_{k} g(m-2 k) V_{3}(k) .
\end{aligned}
$$

Substitute (15) into (17):

$$
\begin{aligned}
Y(m)= & 2 \sum_{k} h(m-2 k) Y_{1}(k) \\
& +2 \sum_{k} g(m-2 k)\left(D_{1} \widehat{\times} W_{1}\right)(k), \\
Y_{1}(m)= & 2 \sum_{k} h(m-2 k) Y_{2}(k) \\
& +2 \sum_{k} g(m-2 k)\left(D_{2} \widehat{\times} W_{2}\right)(k), \\
Y_{2}(m)= & \sum_{k} h(m-2 k) Y_{3}(k) \\
& +\sum_{k} g(m-2 k)\left(D_{3} \widehat{\times} W_{3}\right)(k) .
\end{aligned}
$$

3.2.1. $\partial e(n) / \partial w_{j, k}$ and $\partial e(n) / \partial u_{i}$. According to (11a) and (11b) and (12), $\partial e(n) / \partial w_{j, k}$ should be first given so as to calculate $\widehat{\nabla}_{j}$. To address this problem, this study employs induction. Here, $\partial e(n) / \partial w_{1, i}, \partial e(n) / \partial w_{2, i}$, and $\partial e(n) / \partial w_{3, i}$ are given as follows.

(1) $\partial e(n) / \partial w_{1, i}$. By (16), $\partial e(n) / \partial w_{1, i}$ can be expressed as

$$
\frac{\partial e(n)}{\partial w_{1, i}}=\sum_{m=0}^{N-1} \frac{\partial Y(m)}{\partial w_{1, i}} .
$$

Substitute (18) into above equation:

$$
\begin{aligned}
\frac{\partial e(n)}{\partial w_{1, i}}= & 2 \sum_{m=0}^{N-1} \sum_{k_{0}} h\left(m-2 k_{0}\right) \frac{\partial Y_{1}\left(k_{0}\right)}{\partial w_{1, i}} \\
& +2 \sum_{m=0}^{N-1} \sum_{k_{0}} g\left(m-2 k_{0}\right) \frac{\partial\left(D_{1} \widehat{\times} W_{1}\right)\left(k_{0}\right)}{\partial w_{1, i}} .
\end{aligned}
$$

It is clear that $Y_{1}$ is not correlative with $W_{1}$, so (22) can be rewritten as

$$
\frac{\partial e(n)}{\partial w_{1, i}}=2 \sum_{m=0}^{N-1} g(m-2 i) D_{1}(i)
$$

(2) $\partial e(n) / \partial w_{1, i}$. In the same way, $\partial e(n) / \partial w_{1, i}$ can be expressed by

$$
\frac{\partial e(n)}{\partial w_{2, i}}=\sum_{m=0}^{N-1} \frac{\partial Y(m)}{\partial w_{2, i}} .
$$

Substitute (18) into (23), and

$$
\frac{\partial\left(D_{1} \widehat{x} W_{1}\right)\left(k_{0}\right)}{\partial w_{2, i}}=0 .
$$

Then (24) can be rewritten as

$$
\frac{\partial e(n)}{\partial w_{2, i}}=2 \sum_{m=0}^{N-1} \sum_{k_{0}} h\left(m-2 k_{0}\right) \frac{\partial Y_{1}\left(k_{0}\right)}{\partial w_{2, i}} .
$$


Substitute (19) into (26), and

$$
\frac{\partial Y_{2}\left(k_{1}\right)}{\partial w_{2, i}}=0
$$

So, the final expression of $\partial e(n) / \partial w_{1, i}$ is

$$
\frac{\partial e(n)}{\partial w_{1, i}}=2^{2} \sum_{m=0}^{N-1} \sum_{k_{0}} h\left(m-2 k_{0}\right) g\left(k_{0}-2 i\right) D_{1}(i) .
$$

(3) $\partial e(n) / \partial w_{3, i}$. Similarly, $\partial e(n) / \partial w_{3, i}$ can also be obtained; it is represented by

$$
\begin{aligned}
& \frac{\partial e(n)}{\partial w_{3, i}} \\
& \quad=2^{3} \sum_{m=0}^{N-1} \sum_{k_{0}} h\left(m-2 k_{0}\right) \sum_{k_{1}} h\left(k_{0}-2 k_{1}\right) g\left(k_{1}-2 i\right) D_{2}(i) .
\end{aligned}
$$

From the above, $\partial e(n) / \partial w_{1, i}, \partial e(n) / \partial w_{1, i}$, and $\partial e(n) / \partial w_{3, i}$ have been obtained. By comparing them with each other, the universal expression of $\partial e(n) / \partial w_{j, i}$ can be induced:

$$
\begin{aligned}
& \text { If } j=1 \quad \frac{\partial e(n)}{\partial w_{1, i}}=\sum_{m=0}^{N-1} g(m-2 i) D_{1}(i), \\
& \text { If } j \neq 1 \quad \frac{\partial e(n)}{w_{j i}}=\sum_{m=0}^{N-1} Z_{j}(m, i) D_{j}(i),
\end{aligned}
$$

where $j=1, \ldots, J$ and $i=0, \ldots, 2^{-j} N-1$. Consider

$$
\begin{aligned}
& Z_{j}(m, i) \\
& =2^{j} \sum_{k_{0}} \sum_{k_{1}} \cdots \sum_{k_{j-1}} h\left(m-2 k_{0}\right) \cdots h\left(k_{j-3}-2 k_{j-2}\right) g\left(k_{j-2}-2 i\right) .
\end{aligned}
$$

As $U$ is the same as $W_{j}$ in the adaptation way, we can easily obtain the expression of $\partial e(n) / \partial u_{i}$ :

$$
\frac{\partial e(n)}{\partial u_{i}}=2^{J} \sum_{m=0}^{N-1} V_{J}(m, i) X_{J}(i)
$$

where $i=0, \ldots, 2^{-J} N-1$. Consider

$V_{J}(m, i)$

$=2^{J} \sum_{k_{0}} \sum_{k_{1}} \cdots \sum_{k_{j-1}} h\left(m-2 k_{0}\right) \cdots h\left(k_{J-3}-2 k_{J-2}\right) h\left(k_{J-2}-2 i\right)$.

\subsubsection{Adaptation of $W_{j}$ and $U$}

(1) $W_{j}$

$$
\begin{aligned}
& \text { If } j=1 \quad w_{1 i}=w_{1 i}+2^{2} \mu_{1} e(n) \sum_{m=0}^{N-1} g(m-2 i) D_{1}(i) \\
& \text { If } j \neq 1 \quad w_{j i}=w_{j i}+2^{j+1} \mu_{j} e(n) \sum_{m=0}^{N-1} Z_{j}(m, i) D_{j}(i),
\end{aligned}
$$

where $1 \leq j \leq J, 0 \leq i<2^{-j} N$.
$Z_{j}(m, i)$ is the same meaning as expression above, and $\mu_{j}$ is the step-size of $j$ th level detail signal.

(2) $U$

$$
u_{i}=u_{i}+2^{J+1} \mu_{J} e(n) \sum_{m=0}^{N-1} V_{J}(m, i) X_{J}(i)
$$

where $V_{J}(m, i)$ is also the same meaning as the expression above and $\mu_{J}$ is the step-size of final approximation signal.

\section{MRE Noise Control System}

4.1. Dynamics of MRE. Magneto-rheological elastomers (MREs) are promising smart materials, which consist of magnetically polarizable particles in nonmagnetic solid or gel-like medium $[8,9]$. As a compound of smart magnetorheological (MR) fluid and viscoelastic materials, the MRE combines the salient features of both materials, including controllable stiffness and frequency-dependent viscoelastic behavior, which can be reversibly controlled under external magnetic fields in milliseconds.

The MRE is generally regarded as a viscoelastic material [10], so its dynamic behavior can be described with a complex modulus which depends on vibration frequency and be controllable by external magnetic fields. In order to analyze the mechanical properties of MRE in the magnetic field, the modal of MRE can be simplified to a general kelvin model of viscoelastic material in parallel with an adjustable spring which was controlled by the applied magnetic field, as shown in Figure 4. In the noise control system, as the strain caused by the noise is small, the modal of MRE can be treated as a linear modal, in which the magnetic-induced modulus is adjusted by the applied magnetic field.

As shown in Figure 4, when the MRE is subjected to the applied alternate strain where

$$
\gamma(t)=\gamma_{0} e^{i \omega t}=\gamma_{0}(\cos \omega t+\sin \omega t)
$$

the corresponding stress will become

$$
\tau(t)=G_{0} \gamma+G_{m} \gamma+\eta \dot{\gamma}=\tau_{0} e^{i(\omega t+\delta)} .
$$

According to the definition of complex modulus and (1) and (2), the complex modulus of MRE can be expressed as below:

$$
G(i \omega)=\frac{\tau(t)}{\gamma(t)}=G^{I}(i \omega)+i G^{R}(i \omega),
$$

where $\gamma$ and $\tau$ denote the shear strain and stress of the MRE, $\gamma_{0}$ and $\tau_{0}$ represent amplitudes of strain and stress, respectively, and $G_{0}, G_{m}$, and $\eta$ express the initial modulus, variable modulus, and viscosity coefficient, respectively. $\omega$ is the vibration frequency; $\delta$ is the delayed phase between stress and strain.

The real part of the complex modulus $G^{R}(i \omega)$ is storage modulus which is representing the viscoelastic stiffness of the MRE. The imaginary part $G^{I}(i \omega)$ is loss modulus, and 


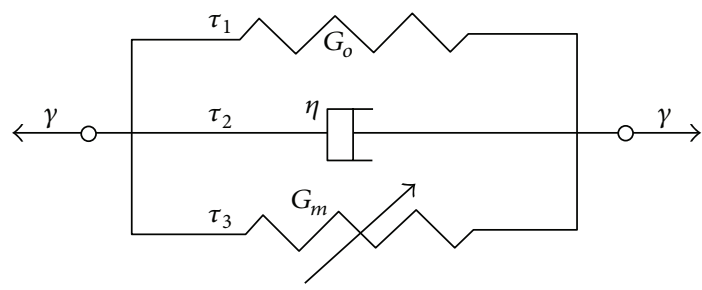

FIGURE 4: The viscoelastic model of MRE.

the ratio of $G^{I}(i \omega)$ to $G^{R}(i \omega)$ refers to the loss factor which is representing the viscoelastic damping and it is shown as

$$
\tan \delta=\frac{G^{I}(i \omega)}{G^{R}(i \omega)} .
$$

According to the corresponding references [11-13], experimental results showed that the storage modulus linearly increases with vibration frequency in most cases, and both the storage modulus and the loss modulus linearly increase with external magnetic field strength in a certain range. Therefore, the storage modulus $G^{R}(i \omega)$ and the loss factor $\tan \delta$ can be approximately expressed by

$$
\begin{gathered}
G^{R}(i \omega)=\alpha_{0}+\alpha_{1} \omega, \\
\tan \delta=\beta_{0},
\end{gathered}
$$

where the coefficients $\alpha_{0}, \alpha_{1}$, and $\beta_{0}$ depend only on the applied magnetic field strength.

4.2. MRE Noise Control System. To test the feasibility of the algorithm stated before, an MRE noise control system is constructed as shown in Figure 5. The system included the MRE, an electromagnetic device (not given in the figure), a noise generation device, and a measurement system. The MRE is made of silicone rubber filled with randomly dispersed carbonyl iron particles. The external magnetic field applied to MRE is generated by a DC electrical source. The measurement system includes $M$ and $L$ sensors which are used to record the excitation and response signals, respectively.

4.3. Simulation Experiment. In order to verify the performance of the algorithm, simulations are investigated in this section. Here, we apply the D-LMS algorithm, DR-LMS algorithm, and LMS algorithm to system identification [6], as shown in Figure 6.

In Figure 6, $x(n)$ is the system input, and $H(z)$ is an unknown system. In this system, the output of adaptive filter is desired to track the output of $H(z), e(n)$ is the identification error, and we also choose $e(n)$ as the criterion to judge the performance of the algorithms.

4.3.1. Linear System with Nonlinear Input. This system can be described by the difference equation as below:

$$
y(n)=0.3 y(n-1)-0.6 y(n-2)+f(x(n)) .
$$

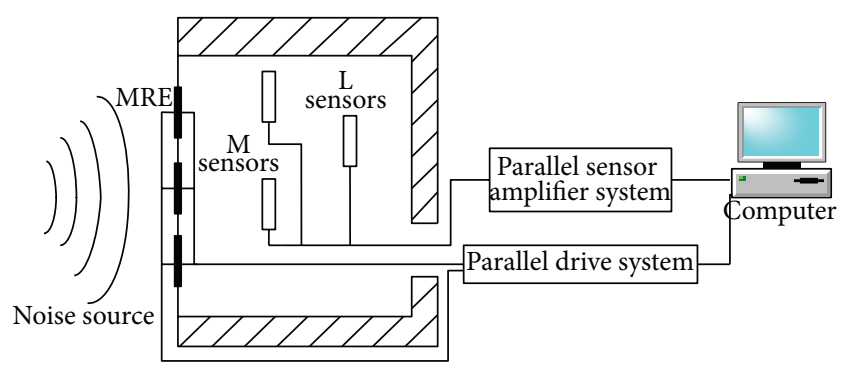

FIGURE 5: A sketch of the MRE noise control system.

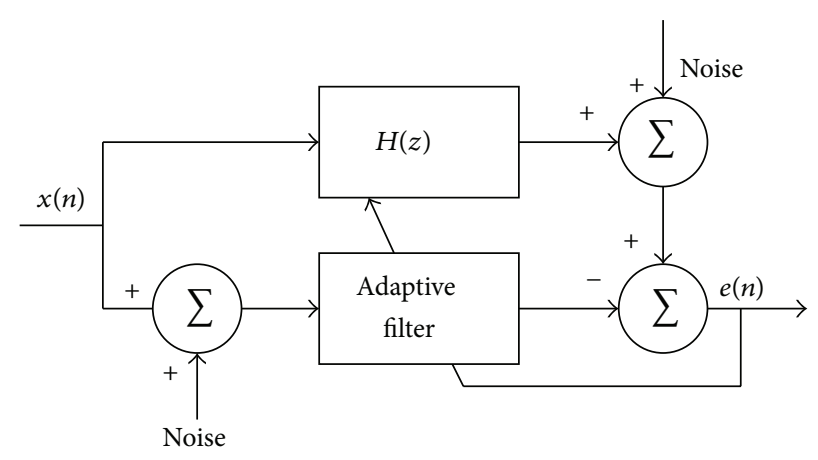

FIgURE 6: System identification.

TABLE 1: Simulation parameters.

\begin{tabular}{lcc}
\hline Algorithm & Length & Step-size \\
\hline LMS & 32 & 0.0003 \\
D-LMS & 32 & 0.1185 \\
DR-LMS & 32 & 0.09 \\
\hline
\end{tabular}

It is a linear dynamic system with a nonlinear input mapping $x(n)$ as the exciting signal. The nonlinear function $f(x)$ is shown as

$$
f(x)=x^{3}+0.3 x^{2}-0.4 x .
$$

Here,

$$
\begin{aligned}
x(n)= & (\sin (2 \pi n / 20)+\sin (2 \pi n / 40) \\
& +\sin (2 \pi n / 50)+\sin (2 \pi n / 250)) \\
& 4.0^{-1} .
\end{aligned}
$$

Table 1 shows the parameters chosen in this simulation. The simulation results are shown in Figures 7 9. The number of the iterations used to update weight vectors is different for different algorithm, while it takes 50 iterations for LMS algorithm but 20 iterations for D-LMS and DR-LMS algorithm. As seen in the figure, there is instability in system identification with LMS algorithm. Table 2 is the mean square error of system identification with different algorithm.

It is obvious that the MSE with D-LMS and DRLMS algorithm are smaller for the same number of samples. 


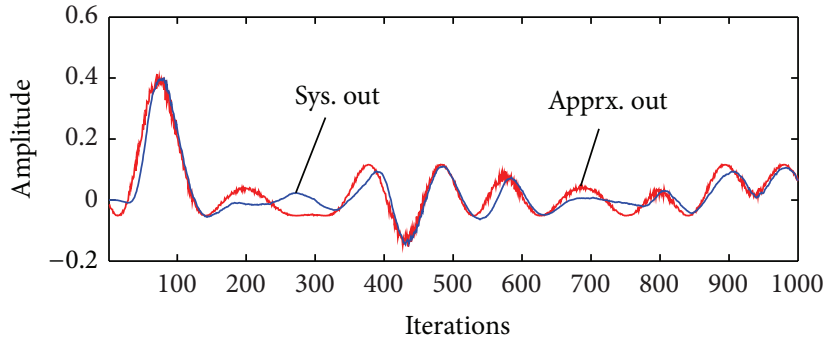

(a)

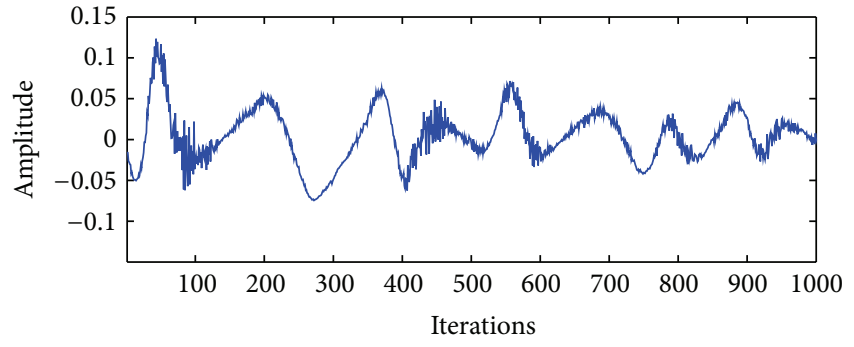

(b)

FIGURE 7: The simulation result with LMS algorithm. (a) Process of identification. (b) Error of identification.

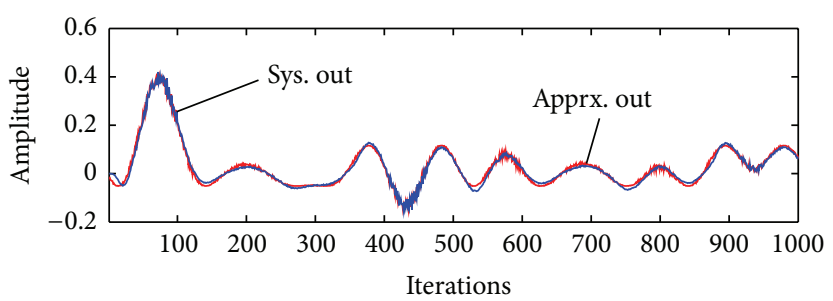

(a)

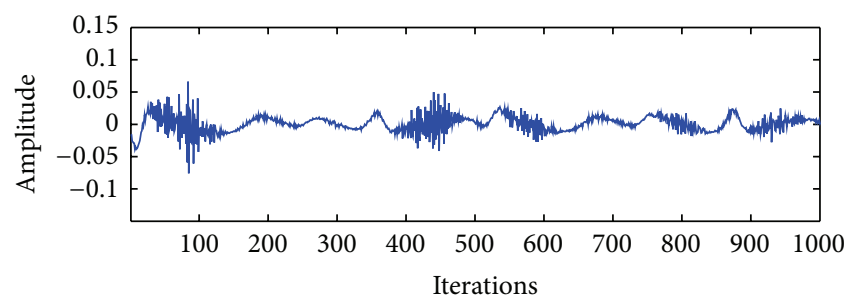

(b)

FIgURE 8: The simulation result with D-LMS algorithm. (a) Process of identification. (b) Error of identification.

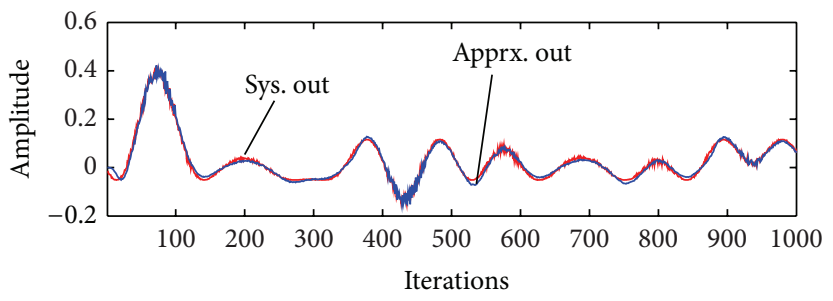

(a)

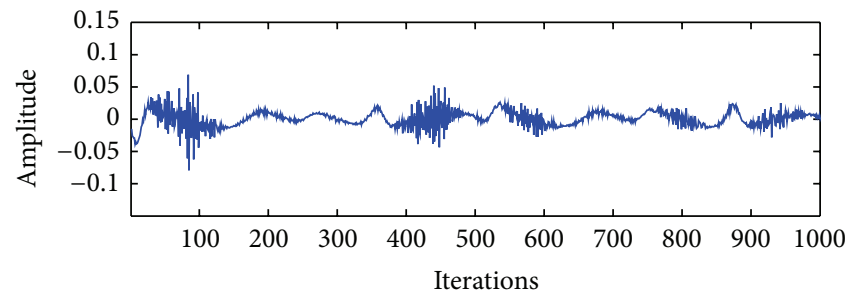

(b)

Figure 9: The simulation result with DR-LMS algorithm. (a) Process of identification. (b) Error of identification.

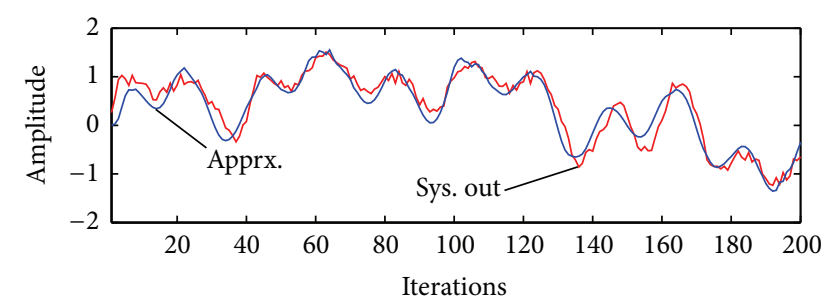

(a)

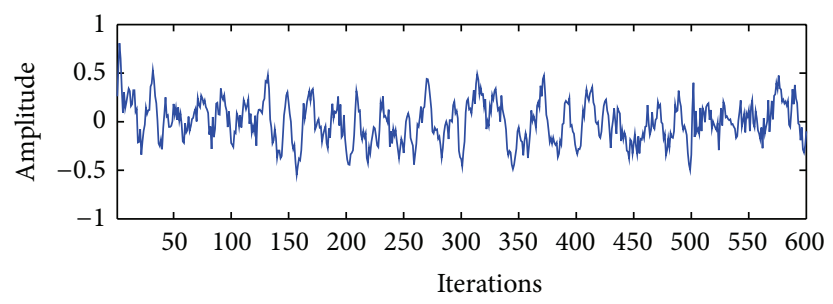

(b)

FIgURE 10: The simulation result with DLMS algorithm. (a) Process of identification. (b) Error of identification.

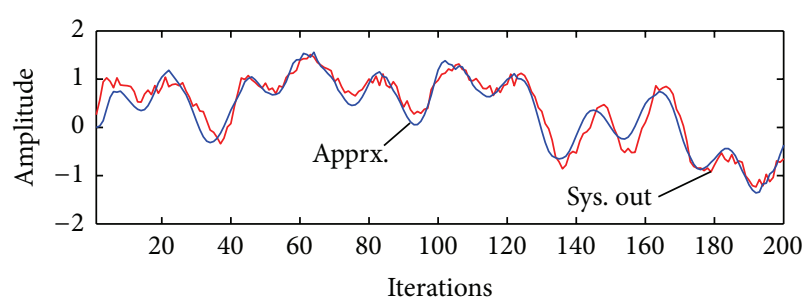

(a)

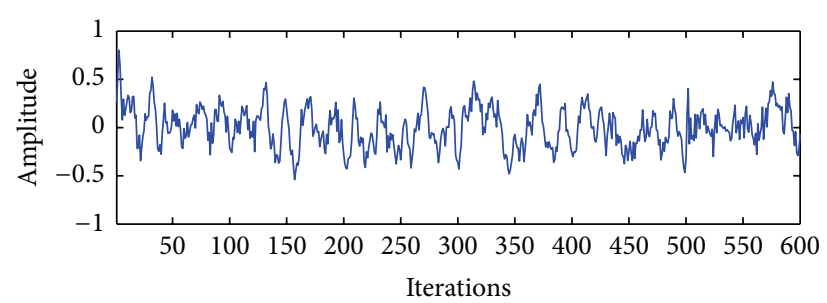

(b)

FIGURE 11: The simulation result with DR-LMS algorithm. (a) Process of identification. (b) Error of identification. 


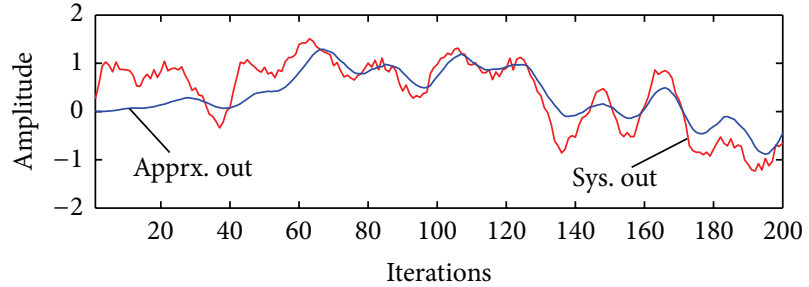

(a)

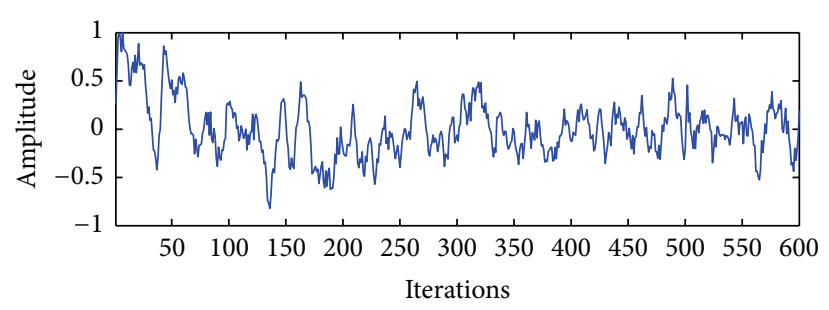

(b)

FIgure 12: The simulation result with LMS algorithm. (a) Process of identification. (b) Error of identification.

TABLE 2: Mean square error (1024 data).

\begin{tabular}{lcc}
\hline LMS & D-LMS & DR-LMS \\
\hline $4.662 \times 10^{-4}$ & $9.0604 \times 10^{-5}$ & $9.216 \times 10^{-5}$ \\
\hline
\end{tabular}

4.3.2. Nonlinear System with Linear Input. This is an example of a nonlinear system which possesses nonlinear dynamics but linear input excitation. The system can be described by a nonlinear difference equation as

$$
y(n)=\frac{y(n-1)}{1+y^{2}(n-1)}+x(n)+w(n)
$$

where $w(n)$ is the noise with uniform distribution and

$$
\begin{aligned}
x(n)= & (\sin (2 \pi n / 20)+\sin (2 \pi n / 50) \\
& +\sin (2 \pi n / 250)+\sin (2 \pi n n / 400)) \\
& \cdot 4^{-1} .
\end{aligned}
$$

The simulation parameters are the same as the previous simulation in Table 1.

As shown in Figure 12, the convergence rate of identification with LMS algorithm is slow, which takes about 70 iterations to obtain stabilization. After about 100 iterations, the mean square error is 0.0628 for 1024 samples. Figures 10 and 11 show that the convergence rates with D-LMS and DRLMS are much faster. After 15 iterations the system can reach stabile state. After about 100 iterations, the mean square errors with D-LMS and DR-LMS are 0.0365 and 0.0355 , respectively, for 1024 samples.

\section{Summary}

In this study, two adaptive algorithms based on Wavelet are proposed and studied. To investigate the performance of the algorithms, the simulation for their application to MRE noise control system is constructed and explored in details. The results indicate that the wavelet adaptive algorithm possesses a good control effectiveness though it requires a great deal of calculation. The implementation of MRE noise control system with DR-LMS algorithm can significantly improve the performance of the control system.

\section{Conflict of Interests}

The authors declare that there is no conflict of interests regarding the publication of this paper.

\section{Acknowledgment}

This paper was supported by the National Natural Science Foundation of China (61373054).

\section{References}

[1] B. Widrow and J. M. McCool, "A comparison of adaptive algorithms based on the methods of steepest descent and random search," IEEE Transactions on Antennas and Propagation, vol. 24, no. 5, pp. 615-637, 1976.

[2] D. F. Marshall, W. K. Jenkins, and J. J. Murphy, "The use of orthogonal transforms for improving performance of adaptive filters," IEEE Transactions on Circuits and Systems, vol. 36, no. 4, pp. 474-484, 1989.

[3] J. C. Lee and C. K. Un, "Performance of transform-domain LMS adaptive digital filters," IEEE Transactions on Acoustics, Speech and Signal Processing, vol. 34, no. 3, pp. 499-510, 1986.

[4] Y. Zhang and P. A. Ioannou, "A new linear adaptive controller: design, analysis and performance," IEEE Transactions on Automatic Control, vol. 45, no. 5, pp. 883-897, 2000.

[5] J. O. Chapa and R. M. Rao, "Algorithms for designing wavelets to match a specified signal," IEEE Transactions on Signal Processing, vol. 48, no. 12, pp. 3395-3406, 2000.

[6] A. Gupta, S. D. Joshi, and S. Prasad, "A new method of estimating wavelet with desired features from a given signal," Signal Processing, vol. 85, no. 1, pp. 147-161, 2005.

[7] Y. Qin, J. Wang, B. Tang, and Y. Mao, "Higher density wavelet frames with symmetric low-pass and band-pass filters," Signal Processing, vol. 90, no. 12, pp. 3219-3231, 2010.

[8] J. D. Carlson and M. R. Jolly, "MR fluid, foam and elastomer devices," Mechatronics, vol. 10, no. 4-5, pp. 555-569, 2000.

[9] M. Kallio, T. Lindroos, and S. Aalto, "The elastic and damping properties of magnetorheological elastomers," Applied Material Research at VTT, vol. 565, pp. 110-120, 2006.

[10] Y. Q. Ni, Z. G. Ying, and Z. H. Chen, "Magneto-rheological elastomer (MRE) based composite structures for micro-vibration control," Earthquake Engineering and Engineering Vibration, vol. 9, no. 3, pp. 345-356, 2010.

[11] J. M. Ginder, M. E. Nichols, L. D. Elie, and J. L. Tardiff, "Magnetorheological elastomers: properties and applications," in Smart Structures and Materials 1999: Smart Materials Technologies, vol. 
3675 of Proceedings of SPIE, pp. 131-138, Newport Beach, Calif, USA, July 1999.

[12] G. Y. Zhou, "Complex shear modulus of a magnetorheological elastomer," Smart Materials and Structures, vol. 13, no. 5, pp. 1203-1210, 2004.

[13] H. Böse, "Viscoelastic properties of silicone-based magnetorheological elastomers," International Journal of Modern Physics B, vol. 21, no. 28-29, pp. 4790-4797, 2007. 

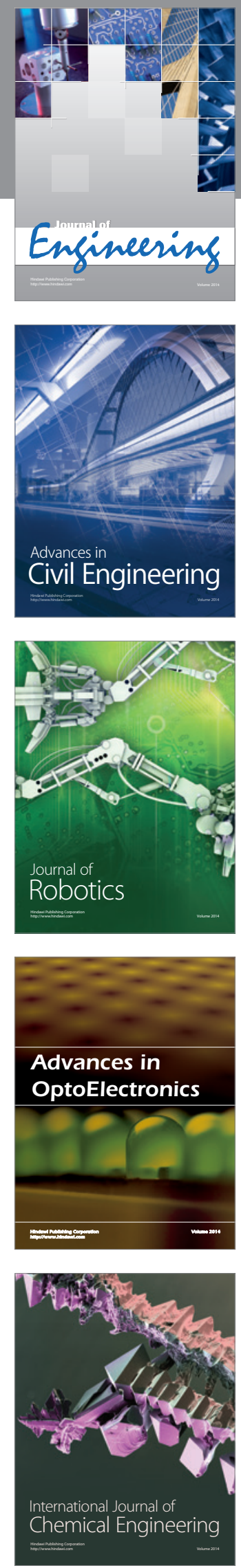

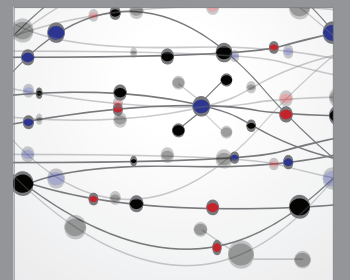

The Scientific World Journal
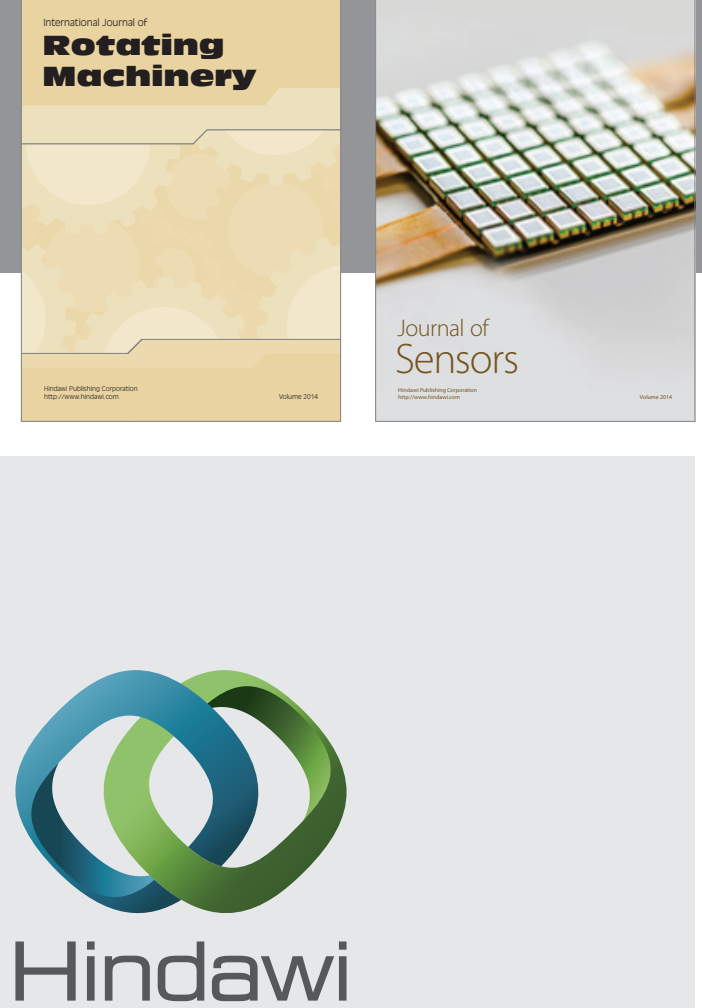

Submit your manuscripts at http://www.hindawi.com
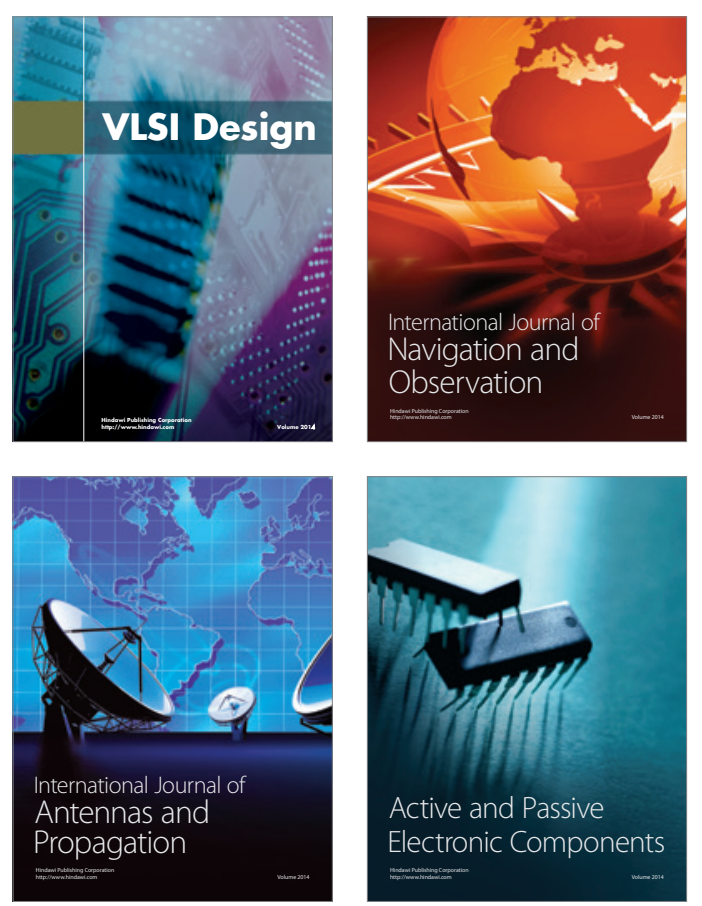
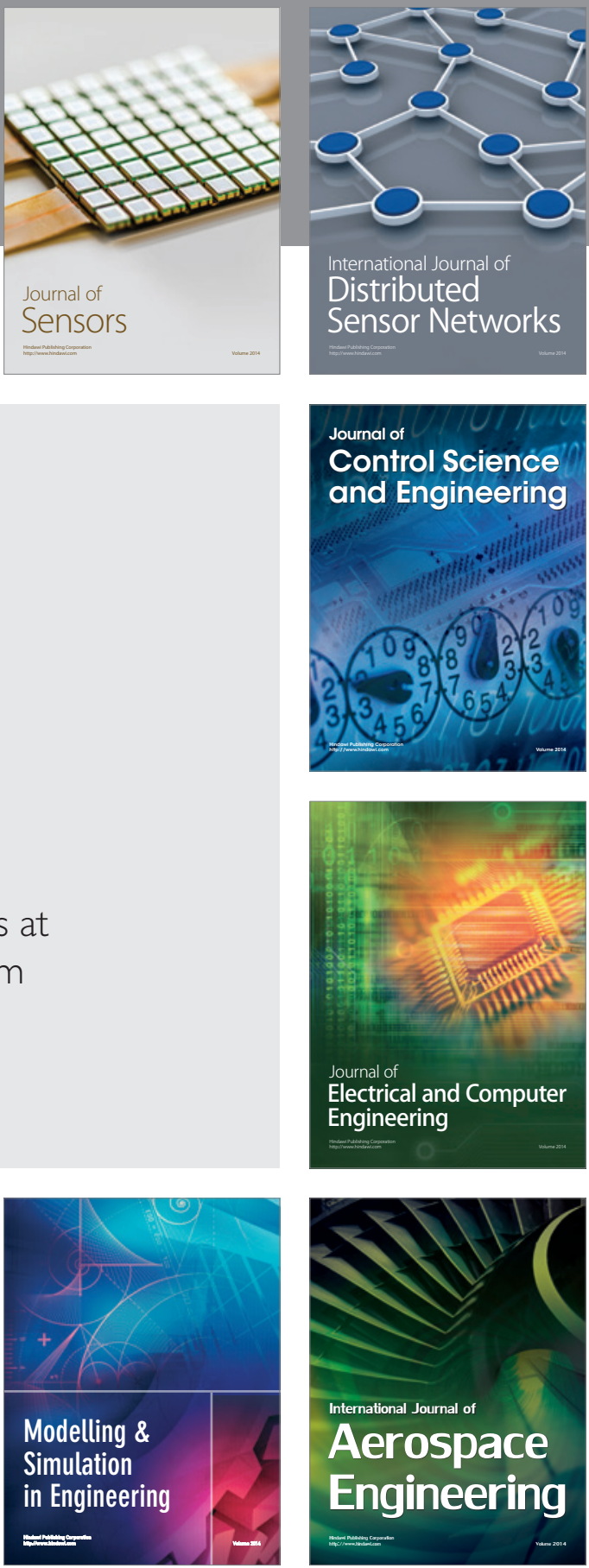

Journal of

Control Science

and Engineering
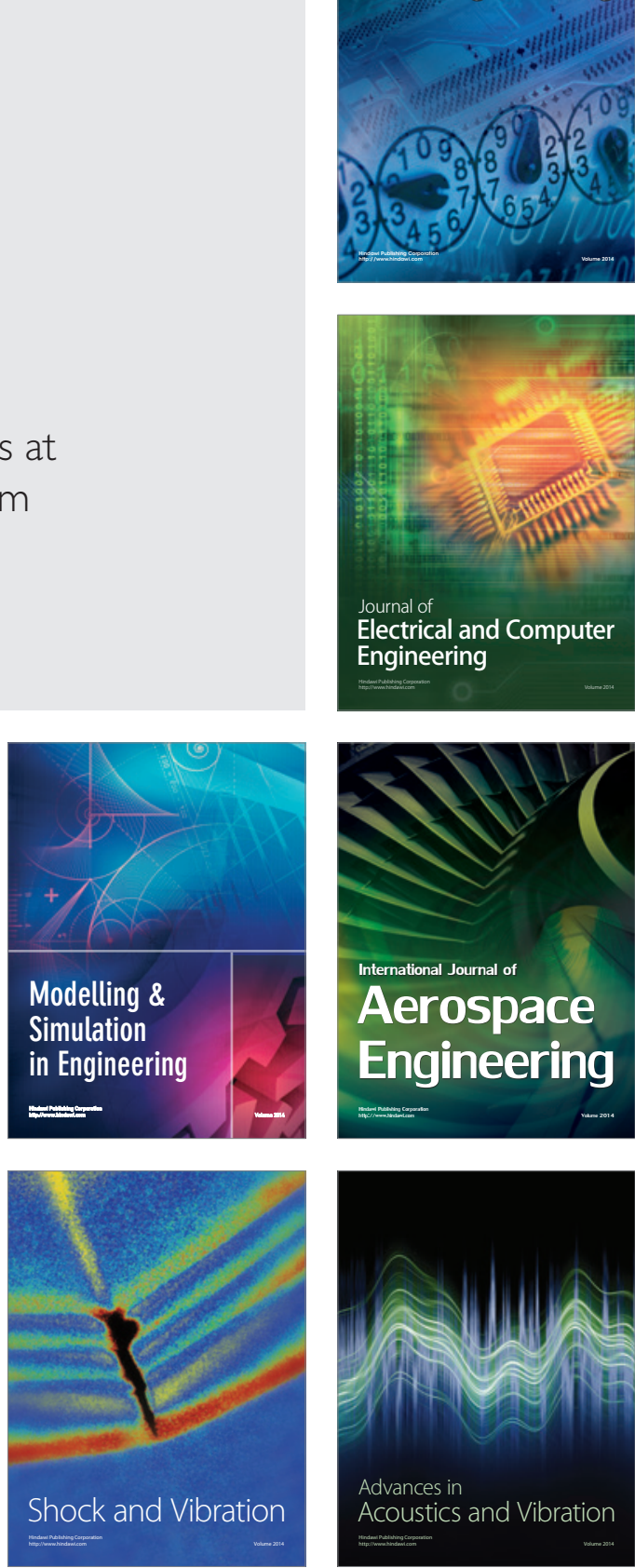